\title{
Preoperative Mean Corpuscular Hemoglobin Concentration, Red Cell Distribution Width As Predictive Markers For Pancreatic Cancer Patients Who Underwent Radical Pancreatomy
}

\section{Dan Wang}

Tumor Hospital of Harbin Medical University

\section{Xiaona Fan}

Tumor Hospital of Harbin Medical University

\section{Lin Fang}

Tumor Hospital of Harbin Medical University

Tianshuo Zhou

Tumor Hospital of Harbin Medical University

Qingwei Li

Tumor Hospital of Harbin Medical University

\section{Wenjing Zhang}

Tumor Hospital of Harbin Medical University

Jinshuang Liu

Tumor Hospital of Harbin Medical University

\section{Ruiyang Li}

Tumor Hospital of Harbin Medical University

\section{Shuling Han}

Tumor Hospital of Harbin Medical University

Zhiwei Li ( $\nabla$ Izhw0451@163.com)

Tumor Hospital of Harbin Medical University

\section{Research}

Keywords: radical pancreatomy, pancreatic cancer, mean corpuscular hemoglobin concentration, red cell distribution width, overall survival, progression-free survival

Posted Date: June 25th, 2020

DOI: https://doi.org/10.21203/rs.3.rs-37763/v1 
License: (c) (i) This work is licensed under a Creative Commons Attribution 4.0 International License. Read Full License 


\section{Abstract}

Objective: To explore the value of mean corpuscular hemoglobin concentration (MCHC) and red cell distribution width (RDW) for overall survival (OS) and progression-free survival (PFS) in patients with pancreatic cancer (PC) who underwent radical pancreatomy.

Background: Inflammatory factors are important factors in promoting the occurrence and metastasis of malignant tumors. RDW and $\mathrm{MCHC}$ are suggested to be relevant to the prognosis of several malignancies. Such as gastric cancer, colon cancer. However, there are few studies to explore the correlation between them and PC.

Methods: This study included 532 patients with PC who underwent radical resection between March 2011 and May 2019. Kaplan-Meier curve method and Cox proportional hazard regression model were applied to analyze prognosis. Correlations between categorical variables were analyzed using Chi squared tests.

Results: Survival curve showed that OS and PFS were significantly favorable for resectable PC patients with high preoperative MCHC $(P=0.02)(P=0.005)$, RDW in the normal range $(P<0.001)(P<0.001)$. Multivariate analysis suggested that $\mathrm{MCHC}$ (HR: $0.612,95 \% \mathrm{Cl}: 0.426-0.879, \mathrm{P}=0.008)$, and RDW (HR: $3.969,95 \% \mathrm{Cl}: 2.952-5.338, \mathrm{P}<0.001)$ were independent prognostic factors for resectable $\mathrm{PC}$ patients. MCHC (HR: 0.657, 95\%Cl: 0.458-0.943, P=0.023), RDW (HR: 3.915, 95\%Cl: 2.923-5.243, P < 0.001) were also independent recurrent factors for resectable PC patients. Correlation analysis showed that $\mathrm{MCHC}$ was related to tumor size, nerve invasion, and anemia-related indicators, meanwhile RDW was connected with carcinoma-embryonic antigen (CEA) and carbohydrate antigen199 (CA19-9).

Conclusion: The preoperative $\mathrm{MCHC}$ and RDW were simple and convenient predictive factors for prognosis and progression of patients with PC.

\section{Introduction}

Pancreatic cancer (PC) is currently the most malignant tumor of the digestive system, which is the third cancer-related death in the United States[1], and possibly turns into the second cancer-related death in the United States by 2030. The median survival time for advanced pancreatic cancer is less than 6 months, and the overall 5-year survival rate is less than $6 \%$ [2]. Surgical resection is still the only way to cure pancreatic cancer [3], however the survival of patients with pancreatic cancer has not been improved significantly, 5 -year survival rate is inferior to $20 \%$ [4]. Although the underlying mechanism of pancreatic cancer is not fully understood, researchers are still searching and identifying useful and easily detected clinical outcome predictors which can instruct clinicians to provide patients with personalized treatment, and prolong progression-free survival (PFS) and overall survival (OS). In recent years, many predictors for pancreatic cancer such as enhanced CT, abdominal ultrasound, postoperative pathology, and tumor markers have been widely used. Although, postoperative pathology is the best way to predict the survival outcome, it is an invasive indicator. Due to individual differences and dietary conditions, tumor markers present some errors in predicting prognosis of patients. Imaging examinations are more costly. Therefore, 
it's urgent to search simple, inexpensive, readily available, and objective biomarkers for diagnosis and prognosis of pancreatic cancer. Inflammatory biomarkers have become research hotspots, and a large number of studies have been conducted worldwide. Erythrocyte-related serological markers are also part of inflammatory biomarkers, and erythrocyte-related inflammatory biomarkers have been shown to be associated with prognosis and recurrence in many cancers. RDW is a hot research topic of erythrocyterelated inflammatory factors in recent years. The study found that RDW was linked to prognosis of many malignancies, including gastric cancer [5], blood cancer [6], kidney cancer [7] and et al. Meanwhile, MCHC was found to be associated with prognosis of oral cavity and lung cancer [8], especially related to the onset of prostate cancer [9]. In addition, recent studies have revealed that patients with high preoperative mean corpuscular volume (MCV) after esophageal surgery achieved a poor prognosis [10], and patients with preoperative red blood cell specific volume $(\mathrm{HCT})<41 \%$ after gastric cancer surgery also had a poor prognosis[11]. However, few studies have explored the relationship between erythrocyte-related serological markers and pancreatic cancer. Therefore our research is dedicated to explore the relationship between both of them.

\section{Material And Methods}

\section{Patients}

A retrospective review on maintained database was performed as much as possible to identify all patients undergoing radical pancreatomy at Harbin Medical University Cancer Hospital from March 2011 to May 2019. Exclusion criteria were as follows: (1) patients who have not undergone routine blood examination before surgery, (2) patients with metastatic disease, (3) patients received any other treatment before enrollment (4) patients who have undergone malignant disease in other organs, and (5) patients who had incomplete/inaccurate medical records. Patients with burns, severe vomiting, diarrhea, chronic carbon monoxide poisoning, cardiac compensatory dysfunction, and hereditary balloon disease affecting MCHC outcomes were excluded [12]. The study was supported by the Ethics Committee of the Harbin Medical University Cancer Hospital. Each patient signed an informed consent form before enrollment.

\section{Data collection}

Clinical and demographic information was obtained from the medical data platform of the Harbin Medical University Cancer Hospital by two independent investigators. Thereafter, all data were collated by the two investigators together. During this procedure, the two independent investigators consulted the medical data platform to resolve any possible bias. These data included the following demographic information: age, sex, body mass index (BMI), hemoglobin (HBG), red blood cell specific volume (HCT), mean corpuscular volume (MCV), mean corpuscular hemoglobin concentration (MCHC), red cell distribution width (RDW), carcinoma-embryonic antigen(CEA), carbohydrate antigen199 (CA19-9). The following clinical information was also collected: date of surgery, tumor location, tumor size, clinical stage, differentiation grade, nerve invasion, vascular invasion, pathological staging. The definition of 
resectable PC referred to the criteria defining resectability status of NCCN Clinical Practice Guidelines in Oncology (3th-edition 2019). All patients with resectable PC underwent RO surgery and had TNM staging based on postoperative pathology. The TNM stage mentioned referred to the Joint Committee on Cancer (8th Edition).

Blood samples for routine blood examination were collected via standard venipuncture of the veins in the antecubital fossae (anterior elbow veins). Routine hematology tests, including HBG, HCT, MCV, MCHC, RDW. $2 \mathrm{ml}$ EDTA anticoagulated peripheral blood and $2 \mathrm{ml}$ plasma anticoagulated by sodium citrate as well as serum samples were obtained before breakfast within 7 days prior to surgery. The samples were promptly centrifuged and processed within two hours. The cell counting of peripheral blood was measured using the SYSMEX XN-9000 full-automated hematology analyzer (Sysmex, Tokyo, Japan).

\section{Follow-up evaluation}

During a mean longitudinal follow-up period of 67 months (from a range of 22 to 103 months) after the surgery, 186 deaths events occurred (58.83\%). Patients were followed every three months until death or the end of the study. The final follow-up evaluation was performed in November 2019. The follow-up data included date of primary resection, date and type of relapse, date of diagnosis of metastatic disease, and date of death. Most follow-up includes physical examination, laboratory tests (blood routine, blood biochemistry, tumor marker CEA, CA199), and imaging studies. The primary end point OS was defined as the time from surgery to death from any cause. PFS and time until recurrence were recorded.

\section{Statistical methods}

Descriptive statistical methods were used to analyze the epidemiological, pathological, and hematological characteristics of the patients. Survival curves were drawn according to the Kaplan-Meier method, survival comparision was executed by Log-rank test. Cox proportional hazard regression model was employed for univariate and multivariate analysis. Statistically significant variables $(P<0.05)$ found in univariate analysis were entered into the Cox regression multivariate model using the backward condition method. A chi-square test was used to analyze the correlation between categorical variables. All tests were double-sided, and statistical significance was inferred at a P value of 0.05 . All statistical analysis was accomplished using SPSS for Windows version25.

\section{Results}

\section{Clinical features of patients}

Of the 532 PC patients included in the study, 317 (59.59\%) were male, and 215 (40.41\%) were female. 451 $(84.77 \%)$ were younger than 65 years old, and most patients had a body mass index in the normal range of $374(70.3 \%) .369(69.36 \%)$ tumors were located on head of pancreas, and adenocarcinoma 500 (93.98\%) was the majority 154 (28.95\%) patients had lymph node involvement. The distribution of TNM stage was $187(35.15 \%)$ of patients in stage I, $325(61.09 \%)$ in stage II, and $20(3.76 \%)$ in stage III (Table 
1). All patients were categorized into the following two groups according to the preoperative MCHC: 481 patients $(90.41 \%)$ in the normal MCHC group and 51 (9.59\%) in the high $\mathrm{MCHC}$ group. According to the preoperative RDW level, 428 (80.45\%) patients had normal RDW and 104 (19.55\%) patients had high RDW. Similarly, there were 6 patients $(1.13 \%)$ in the low MCV group, patients 505 (94.92\%) in the normal MCV group, and 21patients (3.95\%) in the high MCV group.

\section{Survival Analysis}

Survival comparison between patients with $\mathrm{MCHC} \leq 354$ and $\mathrm{MCHC}>354$

The median survival was 12.53 months in high MCHC. The OS and PFS were both significantly higher in the high $\mathrm{MCHC}$ group compared to the normal MCHC group, as was shown in the Kaplan-Meier survival curves in Figure 1. The 5-year OS rate was statistically higher in the MCHC group (5.4 vs. $1.45 \% ; p=0.02$ ) and the PFS proportions were 6.7 vs. $0.7 \%(p=0.005)$ respectively, when comparing the normal against the high MCHC groups.

Survival comparison between patients with $R D W \leq 16.5$ and $R D W>16.5$

The median survival of the RDW normal group was close to the high $\mathrm{MCHC}$ group around 12.13 months. At the same time, the results of RDW were more significant. The log-rank test $p$ values for the differences in OS and PFS between the normal and high RDW groups was demonstrated in Kaplan-Meier survival curves (Figure 1). The 5-year OS rates of the normal and high RDW groups were respectively 1.65 and $0 \%$ $(p<0.001)$ and those of the PFS proportions of the two groups were 1.57 and $0 \%(p<0.001)$.

\section{MCHC and RDW Independently Predicting Prognosis and Recidivation}

In univariate analysis, the preoperative anemic markers of $\mathrm{MCHC}(P<0.05)$ and $\mathrm{RDW}(P<0.05)$ were associated with OS. In addition, other variables, including sex, BMI, postoperative adjuvant chemotherapy, degree of differentiation, nerve invasion, all achieved certain effects on OS (all $P<0.05$, Table 2 ). At the same time, variables including BMI, tumor location, nerve invasion, vascular invasion, HBG, MCHC, RDW had influence on PFS (all $P<0.05$, Table 3). In multivariate analysis, nerve invasion, MCHC, and RDW had a significant effect on OS (all $P<0.05$, table2). Meanwhile, variables integrating degree of differentiation, nerve invasion, $\mathrm{MCHC}$, and RDW achieved an important influence on PFS (all $P<0.05$, Table 3 )

\section{Correlations between MCHC and Clinic Pathological Features}

Table 4 showed the correlations between the MCHC and clinical pathological factors. The MCHC had significant associations with unfavorable clinical pathological factors. Normal MCHC was associated with gender, tumor location (all $P<0.05$, Table 4). Furthermore, nerve invasion, $\mathrm{HCT}$, MCV were significantly associated with normal MCHC (all $P<0.05$, Table 4). Differences from MCHC, RDW were not only related to clinical pathological characteristics, but also had certain correlations with tumor markers CEA and CA19-9 $(P<0.05)$. 


\section{Discussion}

There is growing evidence indicating that tumor-associated inflammation and tumor microenvironment play a more and more important role in the cancer development, progression, metastasis [13], and clinical prognosis [14]. As reported previously, $\mathrm{MCHC}$ and RDW were closely related to the prognosis in several types of cancers. In the present study, we also observed that high $\mathrm{MCHC}$, normal RDW indicated better OS and PFS. Besides, in analyzing the correlation between factors of interest and other clinical pathological variables, $\mathrm{MCHC}$ was higher in patients with the lesion was located on the tail of pancreas, and rarely neurological invasion indicated better survival [15][16]. Therefore, it is reasonable to combine $\mathrm{MCHC}$ and RDW as a simple and convenient marker to enrich the stratification of prognosis in PC patients.

$\mathrm{MCHC}$ means the average red blood cell hemoglobin concentration, is calculated by dividing the red blood cell specific volume (HCT) by the average red blood cell volume (MCV), and is an indicator of the average red blood cell volume [17]. MCHC indirectly reflects the degree of anemia from a quantitative perspective. As we all know, there is increasing evidence that anemia is associated with poor survival rates for a variety of malignancies. Qu found that low MCHC (<335) was associated with unfavorable prognosis of resected lung cancer[8]. Studies also showed that $\mathrm{MCHC}$ was associated with prognosis for oral and head and neck cancers. In his study, the cut-off value of MCHC was decided by ROC curve analysis based on their cohort. However, in this study, the standard value was used as the cut-off value for $\mathrm{MCHC}$ because we believed that it could increase the value of current results and make it universally applicable. Our findings demonstrated that preoperative $\mathrm{MCHC}$ was an independent predictor of OS for pancreatic cancer patients undergoing curative surgical resection. To date, only a few preliminary hypotheses explain why MCHC may affect prognosis. But the mechanism is not clear. This may be related to the following mechanism. At first, Hemoglobin is the main driving force for carrying iron, and anemia can cause excessive iron load. Iron is a trace element involved in a wide range of human metabolism. Iron deficiency and excess can lead to disease. Iron deficiency has been linked to anemia [18], chronic heart failure [19] and other conditions. Excess iron increases the risk of cancer [20]. Even if the excess is physiological, the risk of cancer can increase. [21]. In other words, proper iron consumption achieves a protective effect on the body. The correlation between hematological parameters and serum iron markers may be attributed to changes in iron levels that are to some extent reflected in erythrocyte morphology[22]. Second, Iron overload often accelerates and induces oxidative stress. Oxidative stress is another factor that causes cancer to develop, and serum iron can induce and accelerate this process [23]. Furthermore, the changes of red blood cell parameters indirectly reflect the process of oxidative stress. Third, Hemoglobin is the main driving force for carrying oxygen, and low hemoglobin can cause tissue hypoxia. Hypoxia can promote tumor progression and induce changes in the cell genome to enhance tumor growth and angiogenesis. Hypoxia induces epithelial mesenchyme transformation (EMT) [24], which is critical for cancer progression and metastasis. In addition, hypoxia affects angiogenesis [25]. On the conditions of hypoxia, cancer cells exhibit adaptive metabolic changes. These include conversion of glucose to lactic acid and increased glucose uptake through promotive glucose transporters (GLUTs), a phenomenon known as the Warburg effect [26]. Last, but not the least, Nutrition is another factor leading to the development of disease [27]. Reduced hemoglobin means that patient's nutritional status is 
relatively poor. Increased incidence of complications and increased mortality in patients with nutritional risks before surgery [28]. In clinic, accurate pretreatment staging is essential for treatment decision. Our study found that preoperative $\mathrm{MCHC}$ is an independent prognostic factor for pancreatic cancer, which can affect the prognosis of pancreatic cancer patients. Therefore, as novel and easily obtainable prognostic marker, preoperative $\mathrm{MCHC}$ can be used as a supplement to pathological stages and provide a more accurate prognosis.

RDW, as one of the classical evaluation indexes which can effectively reflect the heterogeneity of erythrocyte volume, and is usually used for evaluation of blood diseases, cardiovascular diseases as well as infectious diseases [29]. In recent years, it has been found that its value is related to the malignant tumor, as a new molecular marker for prognosis and recurrence. Studies have found that an increase in RDW will bring a poor prognosis, which has been confirmed in gastric cancer, colon cancer, liver cancer and other malignant tumor. In this study, we found that Elevated RDW was closely associated with poor prognosis in patients with pancreatic cancer after surgery. In short, preoperative RDW can predict the survival of such patients. The underlying mechanism of cancer development caused by high RDW is generally interpreted as the following. At first, RDW elevation is strongly associated with increase of interleukin- 6 and tumor necrosis factor- $a$, both of which can affect the tumor cell biological behaviors [30]. Pro-inflammatory cytokines are considered to affect iron metabolism and erythropoietin production, shorten the lifespan of red blood cells and induce the release [31]. And then, RDW has been reported as a biomarker in response to inflammation and oxidative stress [24]. Inflammation and oxidative damage foster the progression of cancer, including sustaining proliferative signaling, evading growth suppressors, and activating invasion and metastasis, thus impacting patient survival. Moreover, a higher tumor stage can result in a greater extent of systemic inflammation by the secretion of cytokines and release of tumor-degradation products [32], which then decreases lifetime of red blood cells and increases RDW level. Last, patients with cancer might change their dietary habits or eat less due to insufficient energy intake, which might lead to malnutrition.

To our surprise, we also found that preoperative $\mathrm{MCHC}$ and RDW had an effect on the recurrence of patients with pancreatic cancer after surgery. RDW has been proven to be a marker of tumor recurrence in oral cancer [33]. To date, there are no studies regarding the relationship between $\mathrm{MCHC}$ and cancer recurrence. According to the literature, few inflammatory markers can achieve a monitoring effect on tumor recurrence. And in this article, compared with the relationship between $\mathrm{MCHC}$ and prognosis, the relationship between $\mathrm{MCHC}$ and recurrence is more meaningful. In short, for preoperative $\mathrm{MCHC}$ levels of less than 354, we can take interventions to prolong the relapse time of the disease and prolong the survival of patients. But the mechanism by which $\mathrm{MCHC}$ affects relapses is also unclear and has never been reported. Possible mechanism may be due to inflammation related to malnutrition, immune dysfunction, platelet activation, angiogenesis and cytokine activation [34]. Therefore, preoperative MCHC has a certain predictive effect on the recurrence of pancreatic cancer after surgery. Compared with other monitoring methods, it has the advantages of economy, non-invasiveness and convenience. 
The study also revealed some associations between the MCHC and RDW and clinical pathologic features previously shown to be predictive of worse outcomes. Such as, patients with high MCHC often suffer from tumors in pancreatic body and tail. It is well known that patients with pancreatic body and tail have a better prognosis than the head[16]. Then, patients with high $\mathrm{MCHC}$ are less prone to nerve invasion. Pain is a common clinical symptom of pancreatic cancer, neuropathy is a characteristic of pancreatic cancer, as is well-known that this neuropathy is significantly related to prognosis [15]. In addition, MCHC is associated with HCT, MCV, because they are all indicators of anemia. Although there was no correlation between RDW and clinical pathological parameters, it was significantly correlated with tumor markers. As we all know, tumor markers represent the activity of tumor to some extent, and indirectly reflect the severity and changes of the diseases [35]. RDW combined with CEA and CA19-9 can better predict the prognosis and relapse of patients. Although $\mathrm{MCHC}$ and RDW was associated with a number of variables, the relationship between them and OS was not modified by any other clinical pathological factors, which suggested that both $\mathrm{MCHC}$ and $\mathrm{RDW}$ were stable predictors of prognosis in patients with pancreatic cancer.

This study also had some flaws. At first, this study was a retrospective study. Although we had excluded factors such as blood system diseases and inflammatory diseases that might cause MCHC changes before the study, there were still many unavoidable interference factors which could affect the results of this experiment. Then, this study was single-centered with small sample, leading to biases in sample selection and results analysis. More medical institutions and samples are still needed to further verify this result. Finally, Due to grouping according to the normal range, there was a certain deviation in the two groups of samples, which might have some impacts on our experimental results. In summary, although this study had certain shortcomings, MCHC and RDW had good predictive values for prognosis and recurrence of patients with pancreatic cancer after surgery. At the same time, these indexes achieved the advantages of cheap, convenient, non-invasive, as well as certain research values.

\section{Declarations}

\section{Acknowledgements}

\section{Funding}

This study was sponsored by the National Natural Science Foundation of China (No.81773210); Haiyan Research Fund (No. JJLX2016-01) ; And Graduate Research and Practice Innovation Project.

\section{competing interests}

statement: The authors declare that they have no competing interests

\section{Authors' contributions}

All authors dedicated to the study notion and design. Material preparation, data collection and analysis were accomplished by DW, XNF and LF. The first draft of the manuscript was written by DW. TSZ, WJZ, 
JSL, RYL, SLH collected the date. QWL, ZWL revised the work critically for important intellectual content. All authors commented on previous versions of the manuscript. All authors read and approved the final manuscript.

\section{Ethics approval}

This retrospective study involving human participants meets the ethical standards of the institution and the National Research Council as well as the 1964 Helsinki Declaration and its subsequent amendments or comparable ethical standards. The Human Investigation Committee (IRB) at Harbin Medical University approved the study.The ethical number is: 2019-109-IIIT.

\section{Sample statements consent to participate:}

Informed consent was acquired from all individual participators incorporated in the study.

\section{Data availability statement}

All of the data collected and analysed during the current study are available from the corresponding author on rational demand.

\section{References}

1. Mittler, R., M. Darash-Yahana, Y. S. Sohn, F. Bai, L. Song, I. Z. Cabantchik, P. A. Jennings, J. N. Onuchic and R. Nechushtai . "NEET Proteins: A New Link Between Iron Metabolism, Reactive Oxygen Species, and Cancer." Antioxid Redox Signal (2019) 30(8): 1083-1095.

2. Rahib, L., B. D. Smith, R. Aizenberg, A. B. Rosenzweig, J. M. Fleshman and L. M. Matrisian . "Projecting cancer incidence and deaths to 2030: the unexpected burden of thyroid, liver, and pancreas cancers in the United States." Cancer Res(2014)74(11): 2913-2921.

3. Strobel, O., J. Neoptolemos, D. Jager and M. W. Buchler . "Optimizing the outcomes of pancreatic cancer surgery." Nat Rev Clin Oncol(2019)16(1): 11-26.

4. Abe, T., C. Koi, S. Kohi, K. B. Song, K. Tamura, A. Macgregor-Das, N. Kitaoka, M. Chuidian, M. Ford, M. Dbouk, M. Borges, J. He, R. Burkhart, C. L. Wolfgang, A. P. Klein, J. R. Eshleman, R. H. Hruban, M. I. Canto and M. Goggins. "Gene Variants That Affect Levels of Circulating Tumor Markers Increase Identification of Patients with Pancreatic Cancer." Clin Gastroenterol Hepatol.(2019).

5. Hirahara, N., Y. Tajima, Y. Fujii, S. Kaji, T. Yamamoto, R. Hyakudomi, T. Taniura and Y. Kawabata . "Comprehensive Analysis of Red Blood Cell Distribution Width as a Preoperative Prognostic Predictor in Gastric Cancer." Anticancer Res (2019)39(6): 3121-3130.

6. Ai, L., S. Mu and Y. Hu . "Prognostic role of RDW in hematological malignancies: a systematic review and meta-analysis." Cancer Cell Int (2018)18: 61.

7. Zyczkowski, M., P. Rajwa, E. Gabrys, K. Jakubowska, E. Jantos and A. Paradysz. "The Relationship Between Red Cell Distribution Width and Cancer-Specific Survival in Patients With Renal Cell 
Carcinoma Treated With Partial and Radical Nephrectomy." Clin Genitourin Cancer (2018)16(3): e677e683.

8. Qu, X., T. Zhang, H. Ma, P. Sui and J. J. F. O. Du. "Lower mean corpuscular hemoglobin concentration is associated with unfavorable prognosis of resected lung cancer."(2014)10(14): 2149-2159.

9. Adris, N., A. C. G. Chua, M. W. Knuiman, M. L. Divitini, D. Trinder and J. K. Olynyk. "A prospective cohort examination of haematological parameters in relation to cancer death and incidence: the Busselton Health Study." BMC Cancer(2018)18(1): 863.

10. Yoshida N, Kosumi K, Tokunaga R, et al. Clinical Importance of Mean Corpuscular Volume as a Prognostic Marker After Esophagectomy for Esophageal Cancer: A Retrospective Study[J]. Annals of Surgery, 2018:1.

11. Lin, J. X., J. P. Lin, J. W. Xie, J. B. Wang, J. Lu, Q. Y. Chen, L. L. Cao, M. Lin, R. Tu, C. H. Zheng, C. M. Huang and P. Li. "Preoperative Hematocrit (HCT) is a Novel and Simple Predictive Marker for Gastric Cancer Patients Who Underwent Radical Gastrectomy." Ann Surg Oncol (2019)26(12): 4027-4036.

12. Rajizadeh, A., H. Mozaffari-Khosravi, J. Zavar-Reza and S. M. Shiryazdi. "Comparison of hematological parameters, iron levels, and oxidative stress in women with and without breast cancer: A case- control study." Med J Islam Repub Iran(2017)31: 114.

13. Chan, J. C., D. L. Chan, C. I. Diakos, A. Engel, N. Pavlakis, A. Gill and S. J. Clarke. "The Lymphocyte-toMonocyte Ratio is a Superior Predictor of Overall Survival in Comparison to Established Biomarkers of Resectable Colorectal Cancer." Ann Surg(2017)265(3): 539-546.

14. Gao L , Zhang H, Zhang B , et al. Prognostic value of combination of preoperative platelet count and mean platelet volume in patients with resectable non-small cell lung cancer[J]. Oncotarget, 2017, 8(9).

15. Wang, X., H. Zhang, T. Wang, W. Y. Lau, X. Wang, J. Sun, Z. Yuan and Y. Zhang . "The concept and controversy of retroperitoneal nerve dissection in pancreatic head carcinoma (Review)." Int J Oncol(2015)47(6): 2017-2027.

16. Colloca, G. A., A. Venturino and D. Guarneri . "Retrospective analysis by site of primary tumor of patients with unresectable locally-advanced or metastatic pancreatic adenocarcinoma receiving chemotherapy." Clin Exp Metastasis(2019) 36(6): 519-525.

17. Huang, X. Z., Y. C. Yang, Y. Chen, C. C. Wu, R. F. Lin, Z. N. Wang and X. Zhang . "Preoperative Anemia or Low Hemoglobin Predicts Poor Prognosis in Gastric Cancer Patients: A Meta-Analysis." Dis Markers 2019: 7606128.

18. Stauder, R., P. Valent and I. Theurl. "Anemia at older age: etiologies, clinical implications, and management." Blood(2018) 131(5): 505-514.

19. Yerlikaya, A., M. C. Bulbul, B. Afsar, T. Dagel, G. Aslan, L. Voroneanu, D. Siriopol, A. Covic and M. Kanbay. "Iron in kidney and heart failure: from theory to practice." Int Urol Nephrol(2018) 50(3): 481493.

20. Lagergren, K., K. Wahlin, F. Mattsson, D. Alderson and J. Lagergren. "Haemochromatosis and gastrointestinal cancer." Int J Cancer (2016)139(8): 1740-1743. 
21. Chua, A. C., M. W. Knuiman, D. Trinder, M. L. Divitini and J. K. Olynyk. "Higher concentrations of serum iron and transferrin saturation but not serum ferritin are associated with cancer outcomes." Am J Clin Nutr(2016) 104(3): 736-742.

22. Kautz, L. and E. Nemeth. "Molecular liaisons between erythropoiesis and iron metabolism." Blood(2014) 124(4): 479-482..

23. Miller, K. D., L. Nogueira, A. B. Mariotto, J. H. Rowland, K. R. Yabroff, C. M. Alfano, A. Jemal, J. L. Kramer and R. L. Siegel . "Cancer treatment and survivorship statistics, 2019." CA Cancer J Clin (2019)69(5): 363-385.

24. Zhao, B., Y. Mei, J. Yang and P. Ji . "Erythropoietin-regulated oxidative stress negatively affects enucleation during terminal erythropoiesis." Exp Hematol(2016) 44(10): 975-981.

25. Feng, Y., K. Song, W. Shang, L. Chen, C. Wang, B. Pang and N. J. O. L. Wang . "REDD1 overexpression in oral squamous cell carcinoma may predict poor prognosis and correlates with high microvessel density.(2019)

26. Urata, K., I. Kajihara, H. Miyauchi, T. Mijiddorj, S. Otsuka-Maeda, R. Sakamoto, S. Sawamura, H. Kanemaru, S. Kanazawa-Yamada, K. Makino, J. Aoi, T. Makino, S. Fukushima, Y. Komohara and H. Ihn. "The Warburg effect and tumor immune microenvironment in extramammary Paget's disease: Overexpression of LDHA correlates with immune resistance." J Eur Acad Dermatol Venereol(2019).

27. Dou, L., X. Wang, Y. Cao, A. Hu and L. Li . "Relationship between Postoperative Recovery and Nutrition Risk Screened by NRS 2002 and Nutrition Support Status in Patients with Gastrointestinal Cancer." Nutr Cancer(2020)72(1): 33-40.

28. Peng, H., B. B. Chen, L. L. Tang, L. Chen, W. F. Li, Y. Zhang, Y. P. Mao, Y. Sun, L. Z. Liu, L. Tian, Y. Guo and J. Ma. "Prognostic value of nutritional risk screening 2002 scale in nasopharyngeal carcinoma: A large-scale cohort study." Cancer Sci(2018)109(6): 1909-1919.

29. Sargento, L., A. V. Simoes, S. Longo, N. Lousada and R. Palma Dos Reis. "Red blood cell distribution width is a survival predictor beyond anemia and Nt-ProBNP in stable optimally medicated heart failure with reduced ejection fraction outpatients." Clin Hemorheol Microcirc (2017)65(2): 185-194.

30. Ramsey, M. L., E. Talbert, D. Ahn, T. Bekaiisaab, N. Badi, P. M. Bloomston, D. L. Conwell, Z. Cruzmonserrate, M. Dillhoff and M. R. J. P. Farren. "Circulating interleukin- 6 is associated with disease progression, but not cachexia in pancreatic cancer."(2019)19(1): 80-87.

31. Song, S. Y., C. Hua, D. Dornbors, 3rd, R. J. Kang, X. X. Zhao, X. Du, W. He, Y. C. Ding and R. Meng. "Baseline Red Blood Cell Distribution Width as a Predictor of Stroke Occurrence and Outcome: A Comprehensive Meta-Analysis of 31 Studies." Front Neurol (2019)10: 1237.

32. Schlick, K., T. Magnes, F. Huemer, L. Ratzinger, L. Weiss, M. Pichler, T. Melchardt, R. Greil and A. Egle. "C-Reactive Protein and Neutrophil/Lymphocytes Ratio: Prognostic Indicator for Doubling overall survival Prediction in Pancreatic Cancer Patients." J Clin Med (2019)8(11).

33. Tangthongkum, M., S. Tiyanuchit, V. Kirtsreesakul, P. Supanimitjaroenporn and W. Sinkitjaroenchai . "Platelet to lymphocyte ratio and red cell distribution width as prognostic factors for survival and recurrence in patients with oral cancer." Eur Arch Otorhinolaryngol (2017)274(11): 3985-3992. 
34. Soo R A , Chen Z, Teng R S Y, et al. Prognostic significance of immune cells in non-small cell lung cancer: meta-analysis[J]. Oncotarget, 2018, 9(37).

35. Li X , Li S , Liu L , et al. Effect of Perioperative CEA and CA24-2 on Prognosis of Early Resectable Pancreatic Ductal Adenocarcinoma[J]. Journal of Cancer, 2020, 11(1):9-15.

\section{Tables}

TABLE 1. Baseline patient clinicopathologic characteristics 


\begin{tabular}{|c|c|}
\hline Characteristic & Score(532) \\
\hline \multicolumn{2}{|l|}{ Age } \\
\hline$<65$ & $451(84.77 \%)$ \\
\hline$\geq 65$ & $81(15.23 \%)$ \\
\hline \multicolumn{2}{|l|}{ Gender } \\
\hline Male & $317(59.59 \%)$ \\
\hline Female & $215(40.41 \%)$ \\
\hline \multicolumn{2}{|l|}{ BMI } \\
\hline$<18.5$ & $3(0.56 \%)$ \\
\hline $18.5-25$ & $374(70.30 \%)$ \\
\hline$>25$ & $155(29.14 \%)$ \\
\hline \multicolumn{2}{|l|}{ Tumor location } \\
\hline Caput pancreatis & $369(69.36 \%)$ \\
\hline Pancreatic body and tail & $163(30.64 \%)$ \\
\hline \multicolumn{2}{|l|}{ Pathological type } \\
\hline Adenocarcinoma & $500(93.98 \%)$ \\
\hline Other & $32(6.02 \%)$ \\
\hline \multicolumn{2}{|l|}{ Differentiation } \\
\hline Well-differentiated & $51(9.59 \%)$ \\
\hline Moderately differentiated & $285(53.57 \%)$ \\
\hline Poorly differentiated & 196(36.84\%) \\
\hline \multicolumn{2}{|l|}{$\mathrm{T}$} \\
\hline 1 & $70(13.16 \%)$ \\
\hline 2 & $178(33.46 \%)$ \\
\hline 3 & $284(53.38 \%)$ \\
\hline \multicolumn{2}{|l|}{$\mathrm{N}$} \\
\hline 0 & $378(71.05 \%)$ \\
\hline 1 & $132(24.81 \%)$ \\
\hline 2 & $22(4.14 \%)$ \\
\hline \multicolumn{2}{|l|}{ pTNM stage } \\
\hline I & $187(35.00 \%)$ \\
\hline II & $451(61.00 \%)$ \\
\hline III & $451(4.00 \%)$ \\
\hline Vascular invasion & \\
\hline
\end{tabular}


positive

20(3.76\%)

negative

Nerve invasion

positive

negative

HBG

Low

Normal

High

HCT

Low

Normal

High

MCV

Low

Normal

High

$\mathrm{MCHC}$

Normal

High

RDW

Normal

High

CEA

Normal

High

CA19-9

Normal

High
512(96.24\%)

$140(26.32 \%)$

392(73.68\%)

110(20.68\%)

385(72.37\%)

$37(6.95 \%)$

$140(26.32 \%)$

$359(67.48 \%)$

$33(6.20 \%)$

$6(1.13 \%)$

505(94.92\%)

21(3.95\%)

481(90.41\%)

$51(9.59 \%)$

428(80.00\%)

104(20.00\%)

373(70.11\%)

126(23.68\%)

104(19.55\%)

419(78.76\%)

BMI: Body Mass Index HBG: hemoglobin HCT: red blood cell specific volume MCV: mean corpuscular volume MCHC: mean corpuscular hemoglobin concerntration RDW: Red Cell Distribution Width CEA: carcinomaembryonic antigen CA19-9: Carbohydrate antigen19-9. 
TABLE 2. Cox regression Analysis (Backward Stepwise Elimination) for Overall Survival

\begin{tabular}{|c|c|c|c|c|}
\hline & Univariate analysis & & Mulitivariate analysis & \\
\hline Characteristics & $\mathrm{HR}(95 \% \mathrm{CI})$ & $\mathrm{P}$ & $\mathrm{HR}(95 \% \mathrm{CI})$ & $P$ \\
\hline Age & $0.981(0.718-1.340)$ & 0.902 & & \\
\hline Gender & $1.267(1.004-1.600)$ & 0.047 & $1.148(0.903-1.458)$ & 0.260 \\
\hline BMI & $0.745(0.593-0.936)$ & 0.011 & $0.800(0.620-1.032)$ & 0.085 \\
\hline Tumor location & $1.191(0.927-1.529)$ & 0.172 & & \\
\hline Pathological type & $0.719(0.455-1.135)$ & 0.157 & & \\
\hline Differentiation & $1.430(1.188-1.721)$ & $<0.001$ & $1.387(1.145-1.681)$ & 0.010 \\
\hline $\mathrm{T}$ & $0.836(0.588-1.186)$ & 0.836 & & \\
\hline $\mathrm{N}$ & $1.121(0.910-1.380)$ & 0.282 & & \\
\hline Stage & $0.731(0.382-1.400)$ & 0.149 & & \\
\hline Vascular invasion & $1.229(0.710-2.125)$ & 0.462 & & \\
\hline Nerve invasion & $1.595(1.205-2.110)$ & 0.001 & $1.423(1.073-1.188)$ & 0.014 \\
\hline Postoperative adjuvant therapy & $1.692(1.157-2.475)$ & 0.007 & $1.226(0.834-1.802)$ & 0.301 \\
\hline HBG & $1.079(0.854-1.364)$ & 0.523 & & \\
\hline HCT & $0.981(0.788-1.223)$ & 0.866 & & \\
\hline $\mathrm{MCV}$ & $1.116(0.646-1.927)$ & 0.694 & & \\
\hline $\mathrm{MCHC}$ & $0.667(0.469-0.946)$ & 0.023 & $0.612(0.426-0.879)$ & 0.008 \\
\hline RDW & $3.869(2.907-5.147)$ & $<0.001$ & $3.969(2.952-5.338)$ & $<0.001$ \\
\hline CEA & $1.292(0.985-1.695)$ & 0.064 & & \\
\hline CA19-9 & $1.021(0.771-1.351)$ & 0.884 & & \\
\hline
\end{tabular}

BMI: Body Mass Index HBG: hemoglobin HCT: red blood cell specific volume MCV: mean corpuscular volume MCHC: mean corpuscular hemoglobin concerntration RDW: Red Cell Distribution Width CEA: carcinomaembryonic antigen CA19-9: Carbohydrate antigen19-9

TABLE 3. Cox regression Analysis (Backward Stepwise Elimination) for Progression-free Survival 


\begin{tabular}{|c|c|c|c|c|}
\hline & Univariate analysis & & Mulitivariate analysis & \\
\hline Characteristics & $\mathrm{HR}(95 \% \mathrm{CI})$ & $\mathrm{P}$ & $\mathrm{HR}(95 \% \mathrm{CI})$ & $\mathrm{P}$ \\
\hline Age & $1.002(0.986-1.019)$ & 0.792 & & \\
\hline Gender & $1.079(0.788-1.478)$ & 0.635 & & \\
\hline BMI & $1.018(1.010-1.027)$ & 0.003 & $1.244(0.958-1.614)$ & 0.101 \\
\hline Tumor location & $1.383(1.000-1.913)$ & 0.050 & $1.309(0.926-1.850)$ & 0.127 \\
\hline Pathological type & $0.773(0.455-1.345)$ & 0.362 & & \\
\hline Differentiation & $1.269(0.992-1.623)$ & 0.058 & & \\
\hline $\mathrm{T}$ & $1.165(0.717-1.892)$ & 0.387 & & \\
\hline $\mathrm{N}$ & $1.039(0.764-1.414)$ & 0.806 & & \\
\hline Stage & $1.251(0.910-1.720)$ & 0.169 & & \\
\hline Vascular invasion & $4.203(1.694-10.425)$ & 0.002 & $0.949(0.544-1.655)$ & 0.853 \\
\hline Nerve invasion & $1.436(1.004-2.054)$ & 0.047 & $1.563(1.180-2.072)$ & 0.002 \\
\hline Postoperative adjuvant therapy & $1.308(0.777-2.203)$ & 0.312 & & \\
\hline HBG & $1.363(1.004-1.850)$ & 0.047 & $1.063(0.838-1.349)$ & 0.615 \\
\hline HCT & $1.280(0.956-1.713)$ & 0.098 & & \\
\hline $\mathrm{MCV}$ & $1.443(0.785-2.654)$ & 0.238 & & \\
\hline $\mathrm{MCHC}$ & $0.457(0.262-0.799)$ & 0.006 & $0.657(0.458-0.943)$ & 0.023 \\
\hline RDW & $1.878(1.314-2.684)$ & 0.001 & $3.915(2.923-5.243)$ & $<0.001$ \\
\hline CEA & $1.298(0.898-1.876)$ & 0.165 & & \\
\hline CA19-9 & $0.954(0.638-1.428)$ & 0.820 & & \\
\hline
\end{tabular}

BMI: Body Mass Index HBG: hemoglobin HCT: red blood cell specific volume MCV: mean corpuscular volume MCHC: mean corpuscular hemoglobin concerntration RDW: Red Cell Distribution Width CEA: carcinomaembryonic antigen CA19-9: Carbohydrate antigen19-9

TABLE 4.The correlation of clinicopathological characteristics of 532 patients with resectable PC between normal and high groups in terms of MCHC and RDW. 


\begin{tabular}{|c|c|c|c|c|c|c|}
\hline \multirow[b]{2}{*}{ Characteristics } & \multicolumn{2}{|c|}{$\mathrm{MCHC}$} & \multirow[b]{2}{*}{$\mathrm{P}$} & \multicolumn{2}{|c|}{ RDW } & \multirow[b]{2}{*}{$\mathrm{P}$} \\
\hline & $\begin{array}{l}\text { Normal } \\
(n=481)\end{array}$ & $\begin{array}{l}\text { High } \\
(\mathrm{n}=51)\end{array}$ & & $\begin{array}{l}\text { Normal } \\
(n=428)\end{array}$ & $\begin{array}{c}\text { High } \\
(\mathrm{n}=104)\end{array}$ & \\
\hline Age & & & 0.123 & & & 0.388 \\
\hline$<65$ & $404(75.94)$ & $47(8.83)$ & & $360(67.67)$ & $91(17.11)$ & \\
\hline$\geq 65$ & $77(14.47)$ & $4(0.75)$ & & $68(12.78)$ & $13(2.44)$ & \\
\hline Gender & & & 0.047 & & & 0.044 \\
\hline Male & $280(52.63)$ & $37(6.95)$ & & $246(46.24)$ & $71(13.35)$ & \\
\hline Female & $201(37.78)$ & $14(2.63)$ & & $182(34.21)$ & $33(6.2)$ & \\
\hline BMI & & & 0.235 & & & 0.030 \\
\hline$<18.5$ & $3(0.56)$ & $0(0.00)$ & & $1(0.19)$ & $2(0.38)$ & \\
\hline $18.5-25$ & $333(62.59)$ & $41(7.71)$ & & 295(55.45) & $79(14.85)$ & \\
\hline$>25$ & $145(27.26)$ & $10(1.88)$ & & $132(24.81)$ & $23(4.32)$ & \\
\hline Tumor location & & & 0.015 & & & 0.497 \\
\hline Caput pancreatis & $326(61.28)$ & $43(8.08)$ & & $294(55.26)$ & $75(14.10)$ & \\
\hline Pancreatic body and tail & $155(29.14)$ & $8(1.5)$ & & 134(25.19) & $29(5.45)$ & \\
\hline Pathological type & & & 0.069 & & & 0.207 \\
\hline Adenocarcinoma & $455(85.53)$ & $45(8.46)$ & & $405(76.12)$ & $95(17.86)$ & \\
\hline Other & $26(4.89)$ & $6(1.13)$ & & $23(4.32)$ & $9(1.69)$ & \\
\hline Differentiation & & & 0.266 & & & 0.16 \\
\hline Well-differentiated & $43(8.08)$ & $8(1.5)$ & & $43(8.08)$ & $8(1.5)$ & \\
\hline Moderately differentiated & $258(48.5)$ & $27(5.08)$ & & $229(43.05)$ & $56(10.53)$ & \\
\hline Poorly differentiated & $180(33.83)$ & $16(3.01)$ & & 156(29.32) & $40(7.52)$ & \\
\hline $\mathrm{T}$ & & & 0.008 & & & 0.218 \\
\hline 1 & $59(11.09)$ & $11(2.07)$ & & $51(9.59)$ & $19(3.57)$ & \\
\hline 2 & $155(29.14)$ & $23(4.32)$ & & $144(27.07)$ & $34(6.40)$ & \\
\hline 3 & $267(50.19)$ & $17(3.2)$ & & $233(43.8)$ & $51(9.59)$ & \\
\hline $\mathrm{N}$ & & & 0.361 & & & 0.719 \\
\hline 0 & $344(64.66)$ & $34(6.39)$ & & $307(57.71)$ & $71(13.35)$ & \\
\hline 1 & $119(22.37)$ & $13(2.44)$ & & 103(19.37) & $29(5.45)$ & \\
\hline 2 & 18(3.38) & $4(0.75)$ & & 18(3.38) & $4(0.75)$ & \\
\hline pTNM stage & & & 0.220 & & & 0.847 \\
\hline I & $161(30.26)$ & $26(4.89)$ & & $148(27.82)$ & $39(7.33)$ & \\
\hline II & $303(56.95)$ & $22(4.14)$ & & $264(49.62)$ & $61(11.47)$ & \\
\hline
\end{tabular}


III

Vascular invasion

Positive

Negative

Nerve invasion

Positive

Negative

HBG

Low

Normal

High

HCT

Low

Normal

High

MCV

Low

Normal

High

CEA

Normal

High

CA19-9

Normal

High
17(3.2)

3(0.56)

18(3.38) 2(0.38)

463(87.03)

49(9.21)

0.032

133(25.0) 7(1.32)

348(65.41)

44(8.27)

0.659

97(18.23)

13(2.44)

350(65.79)

35(6.58)

34(6.39)

3(0.56)

0.002

117(21.99) 23(4.32)

331(62.22) 28(5.26)

33(6.2)

28(5.26)

0.046

$4(0.75)$

2(0.38)

456(85.71)

$49(9.21)$

21(3.95)

$0(0)$

0.13

340(63.91) 31(5.83)

109(20.49) 16(3.01)

0.725

95(17.86) 9(1.69)

378(71.05) 41(7.71)
16(3.08)

4(0.75)

0.875

112(21.05) 28(5.26)

316(59.40) 76(14.29)

0.07

80(15.04) 30(5.64)

317(59.59) 68(12.78)

31(5.83) 6(1.13)

0.177

106(19.92) 34(6.39)

293(55.08) 66(12.41)

29(5.46) $\quad 4(0.75)$

0.042

3(0.56) 3(0.56)

411(77.26) 94(17.67)

14(2.63) $\quad 7(1.32)$

0.001

302(56.77) 69(12.97)

95(17.86) 30(5.61)

0.001

96(18.05) 8(1.50)

325(61.09) 94(17.67)

BMI: Body Mass Index HBG: hemoglobin HCT: red blood cell specific volume MCV: mean corpuscular volume MCHC: mean corpuscular hemoglobin concerntration RDW: Red Cell Distribution Width CEA: carcinomaembryonic antigen CA19-9: Carbohydrate antigen19-9

Figures 

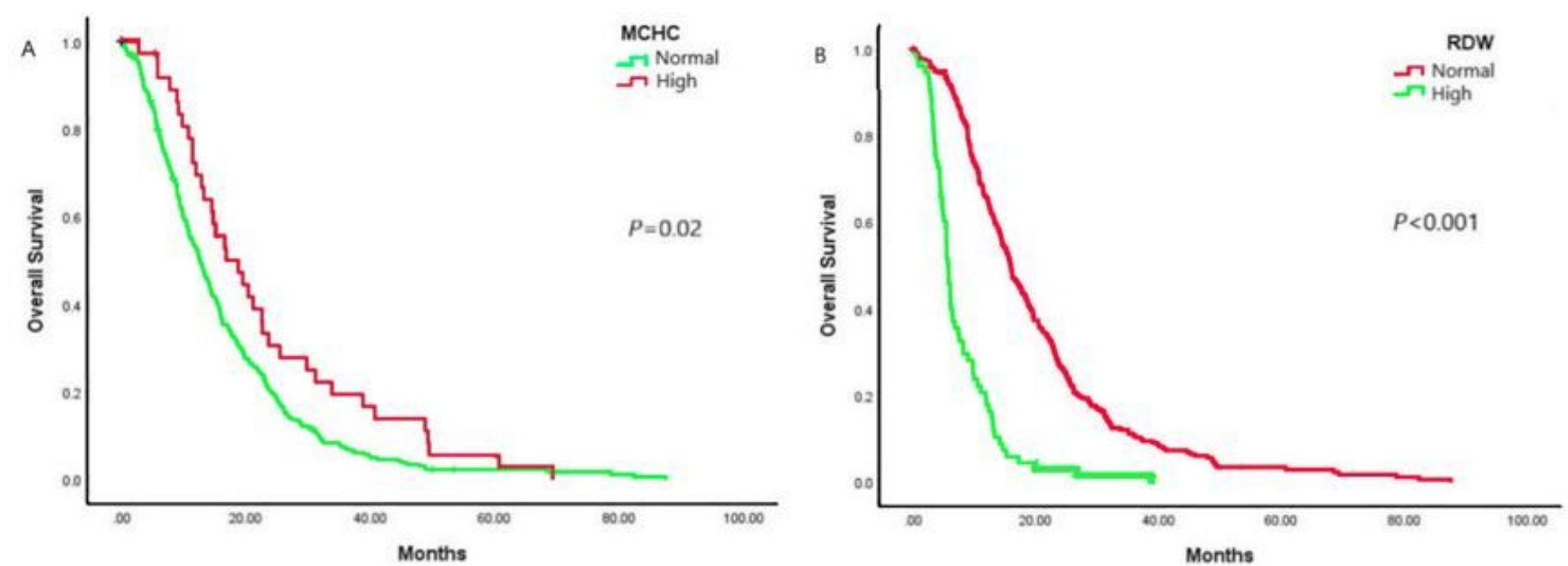

No. at Risk $\begin{array}{lllllll}\text { Normal MCHC } & 189 & 187 & 43 & 8 & 2 & 2 \\ \text { High MCHC } & 30 & 35 & 29 & 5 & 2 & 0\end{array}$

No. at Risk

$\begin{array}{lllllll}\text { Normal RDW } & 189 & 150 & 70 & 13 & 4 & 2 \\ \text { High RDW } & 30 & 72 & 2 & 0 & 0 & 0\end{array}$
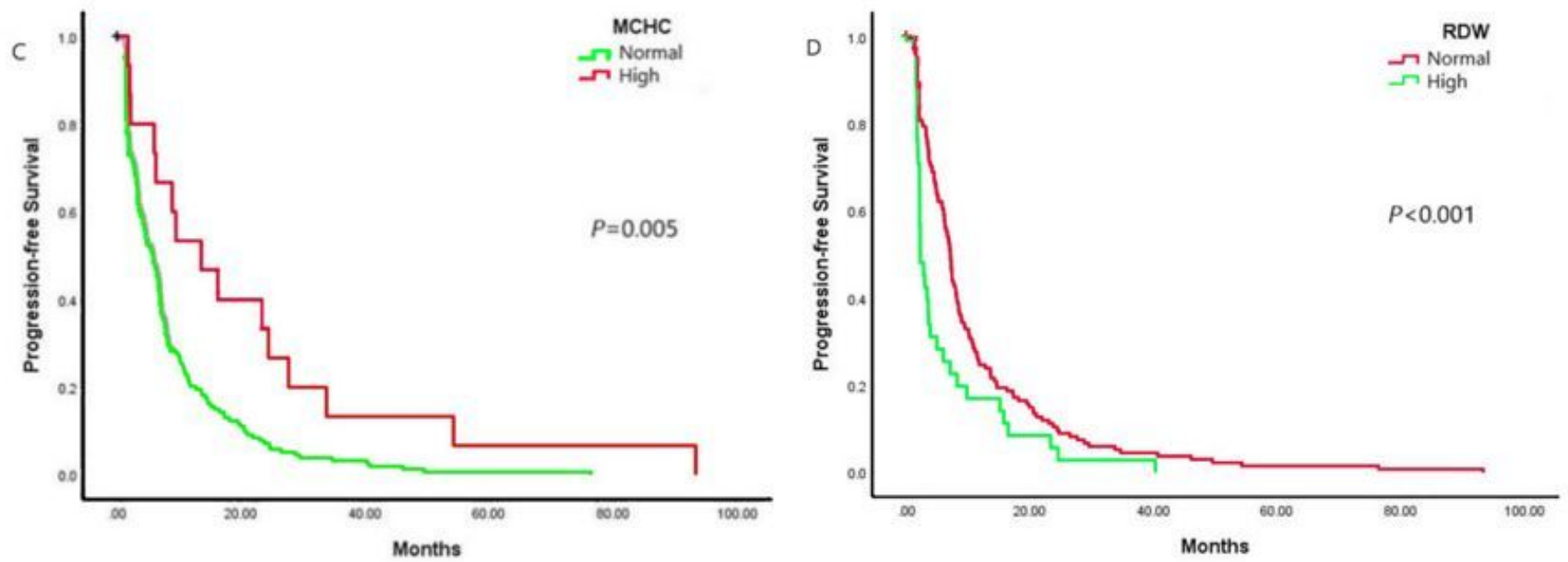

No. at Risk

$\begin{array}{llllll}\text { Normal MCHC } & 321 & 136 & 12 & 4 & 1 \\ \text { High MCHC } & 33 & 9 & 4 & 1 & 0\end{array}$

No, at Risk

$\begin{array}{lllllll}\text { Normal RDW } & 291 & 108 & 14 & 4 & 1 & 1\end{array}$

$\begin{array}{lllllll}\text { High RDW } & 63 & 37 & 2 & 1 & 0 & 0\end{array}$

\section{Figure 1}

Kaplan-Meier survival curves stratified by MCHC and RDW in $532 \mathrm{PC}$ patients underwent radical pancreatomy. A. Kaplan-Meier curves for OS by MCHC, $P=0.02$. B. Kaplan-Meier curves for OS by RDW, $P<0.001$. C. Kaplan-Meier curves for PFS by MCHC. D. Kaplan-Meier curves for PFS by RDW. P values were determined using the log-rank test 